Ann. Zootech., I974, 23 (3), 313-323.

\title{
CROISSANCE RELATIVE DES DIFFÉRENTS TISSUS, ORGANES ET RÉGIONS CORPORELLES DES TAURILLONS FRISONS, DURANT LA PHASE D'ENGRAISSEMENT DE 9 A 15 MOIS
}

\author{
J. ROBELIN, Y. GEAY et C. BÉRANGER \\ Laboratoire de la Production de Viande, \\ Centre de Recherches de Clermont-Ferrand, I. N. R. A., \\ Theix, 63110 Beaumont
}

\section{RÉSUMÉ}

Cette étude a été réalisée à partir de 65 jeunes bovins mâles entiers de race Frisonne, abattus entre 9 et I $_{5}$ mois. Nous avons décrit, en utilisant l'équation d'allométrie de HuXLEY, la croissance relative des principaux composants de l'animal.

La plupart des organes ont un coefficient d'allométrie par rapport au poids vif vide inférieur à $I$, tandis que celui de l'ensemble des dépôts adipeux est voisin de 2, celui de la carcasse légèrement supérieur à $\mathbf{I}$. L'ordre de croissance relative des différents tissus de la carcasse est comparable à celui qui a déjà été observé chez d'autres catégories de bovins et chez d'autres espèces : squelette $(b=0,76)$, muscles $(b=0,92)$, dépôts adipeux de la carcasse $\left(b=1,5^{8}\right)$. Parmi ces derniers, ce sont les dépôts sous-cutanés qui ont la plus forte croissance relative $(b=2,5 \mathrm{I})$. En ce qui concerne la croissance relative des différentes régions de la carcasse, nous avons mis en évidence que chez le bovin mâle entier, la part des régions postérieure et dorsale, à tendance à diminuer pendant l'engraissement, de 9 à $\mathbf{I} 5$ mois, tandis que celle des régions antérieure et abdominale tend à augmenter.

\section{INTRODUCTION}

Les études récentes réalisées en France sur la croissance relative des animaux domestiques ont été effectuées sur le Mouton (BENEVENT, I97I) et le Lapin (VEZINHET et al., I972). En ce qui concerne les bovins, nos conceptions reposent encore généralement sur les résultats de Moulton et al. (I922) et de Hammond et de son École (PAALsON, I955). D'après ces auteurs, il existe des vagues de croissance partant des régions distales de l'animal et se rejoignant dans les derniers stades du développement au niveau des reins. I1 est possible cependant, comme l'ont montré LuiTingH (I962), ButTERFielid (I963) et SEEBECK et TUlLOH (I968, $a$ et $b$ ) que des techniques de dissection séparant les régions ventrales des régions dorsales ne conduisent pas à 
des conclusions identiques, notamment après la période du jeune âge. En outre, la plupart des études précédentes portant sur la croissance des bovins ont été réalisées sur des animaux mâles castrés; or, les androgènes dont la production devient importante à la puberté, agissent sur le développement relatif des différentes régions, notamment au niveau du collier (BRADFIELD, I967). Enfin, la plupart des études réalisées sur les bovins comportaient un nombre restreint d'animaux n'appartenant pas toujours à la même race.

Cette étude a pour objet de décrire l'évolution pondérale des différentes régions corporelles, des différents tissus de la carcasse (os, muscles, dépôts adipeux), et des principaux organes du jeune bovin mâle entier de race Frisonne, au cours de la période d'engraissement de 9 à I5 mois.

\section{MATÉRIEL E'T MÉTHODES}

\section{Animaux}

Nous avons utilisé des données relatives à la dissection de 65 taurillons Frisons engraissés ‘de 9 d̀ I 5 mois au cours de deux expériences, concernant l'influence du mode d'élevage antérieur, ou du niveau d'alimentation pendant la phase d'engraissement, sur les performances des animaux.

Dans la première expérience, deux lots de 23 animaux ont été élevés différemment jusqu'à l'àge de 9 mois. Les animaux du lot I ont été sevrés précocement à l'âge de 3 mois, puis ont reçu une ration constituée essentiellement d'aliments concentrés, en stabulation entravée, jusqu'au début de la période d'engraissement. Les animaux du lot II ont été sevrés à 8 mois après avoir été élevés au pâturage à l'herbe et au lait. Les animaux des deux lots ont ensuite reçu la même alimentation pendant la période d'engraissement. Afin de mesurer l'évolution de la composition corporelle au cours de cette période, on a procédé à l'abattage de 8 animaux par lot à 9 mois, 7 animaux par lot à $\mathbf{1} 2$ mois, et 8 à l'âge de 15 mois.

Au cours de la seconde expérience, Ig animaux ont reçu la même alimentation jusqu'à l'âge de 9 mois ; 7 ont été abattus à cet âge et les $\mathbf{I} 2$ autres abattus à $\mathbf{1} 5$ mois ont été répartis en deux lots de 6 qui ont reçu le même aliment mais en quantités différentes jusqu'à l'abattage. Dans chacune des deux expériences, les animaux abattus à 9 ou I 2 mois ont été choisis de façon à ce que la moyenne et la variance du poids vif soient identiques dans les deux populations d'animaux, ceux qui devaient être abattus, et ceux qui étaient destinés à poursuivre leur croissance.

\section{Mesures (tabl. I)}

Le poids vif de l'animal mesuré juste avant l'abattage pouvant être faussé par les variations du contenu digestif, on a estimé ce poids au jour de l'abattage par extrapolation de la courbe de croissance des animaux, établie à partir de pesées bimensuelles. Le poids vif vide a été calculé

TABLEAU I

Caractéristiques des animaux

\begin{tabular}{c|c|c|c|c}
\hline \hline & Race & $\begin{array}{c}\text { Age } \\
\text { moyen } \\
(\mathbf{j})\end{array}$ & $\begin{array}{c}\text { Poids vif } \\
(\mathrm{kg})\end{array}$ & $\begin{array}{c}\text { Poids de la } \\
\text { carcasse chaude } \\
(\mathrm{kg})\end{array}$ \\
\hline & & & & \\
\hline 14 & Frisons & 282 & $314,0 \pm 39,9$ & $164,7 \pm 25,6$ \\
28 & Frisons & 375 & $444,3 \pm 21,6$ & $245,1 \pm 14,5$ \\
& Frisons & 452 & $521,1 \pm 28,6$ & $290,6 \pm 20,8$ \\
\hline
\end{tabular}


par différence entre le poids réel de l'animal juste avant l'abattage et le poids du contenu digestif.

On a pesé les principaux organes (tube digestif, cœur, foie, poumons, reins...), les différents dépôts adipeux du "cinquième quartier " (périrénaux, péricardiaques, mésentériques, péritonéaux), la tête, le cuir et les os canons (métacarpe et métatarse). La carcasse, sans rein ni gras périrénal, a été pesée chaude.

Après $4^{8}$ heures de ressuyage à la température de $4^{\circ} \mathrm{C}$, la demi carcasse droite (côté sans queue) a été disséquée au couteau de boucher, dans une salle dont la température ambiante était de $10^{\circ} \mathrm{C}$. On a séparé et pesé les différents tissus (os, muscles, dépôts adipeux sous-cutanés, internes et intermusculaires), et un certain nombre de muscles (tabl. 5). Sur 5I animaux (I6 à l'âge de 9 mois, I 8 à I 2 mois, I 7 à I 5 mois), la demi carcasse gauche a fait l'objet d'une découpe bouchère en plusieurs morceaux, définie par Charlet et Dumont (1953) (fig. I). Afin de faciliter la compréhension du texte, nous avons utilisé une dénomination des morceaux correspondant à leur localisation anatomique :

- collier : région cervicale ;

- paleron : membre antérieur ;

— cuisse : membre postérieur ;

- train de côtes : région dorsale ;

- filet et contre filet : région lombaire ;

- rumsteak : région sacrée ;

- plats de côtes, bavettes et pis : régions thoracique et abdominale.

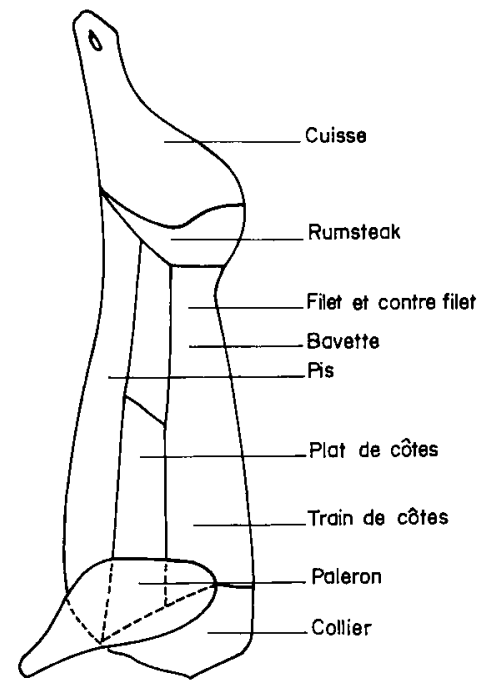

Fig. I. - Découpe française du bouf (d'après P. Charlet et B. L. Dumont, I955)

Méthodes de calculs, signification des mesures

Pour décrire la croissance relative des différents tissus ou organes, nous avons utilisé l'équation d'allométrie de Huxley (I932). La population des animaux étant bien répartie entre $229 \mathrm{~kg}$ et $596 \mathrm{~kg}$ de poids vif (fig. 2), nous avons pu établir l'équation de régression pour les 65 animaux :

$$
\log \mathrm{Y}=b \log \mathrm{X}+a
$$

Dans cette relation, $\mathrm{Y}$ est le poids de la région, de l'organe ou du tissu considéré, $\mathrm{X}$ le poids de l'ensemble de référence, " $a$ " une constante, " $b$ " peut être assimilé au coefficient d'allométrie de $\mathrm{Y}$ par rapport à $\mathrm{X}$, à condition que la relation entre $\log \mathrm{X}$ et $\log \mathrm{X}$ soit effectivement linéaire. Pour vérifier cette linéarité, nous avons effectué un ajustement à une courbe du second degré en " $\log \mathrm{X}$ ", et nous avons comparé par un test de $\mathrm{F}$, les valeurs des variances résiduelles relatives aux deux équations (linéaire et curvilinéaire). Dans le cas où $F$ dépassait la valeur $F(o$, oor), nous avons établi un ajustement à deux droites de régression par la méthode décrite par CANTIER et al. (1969). 


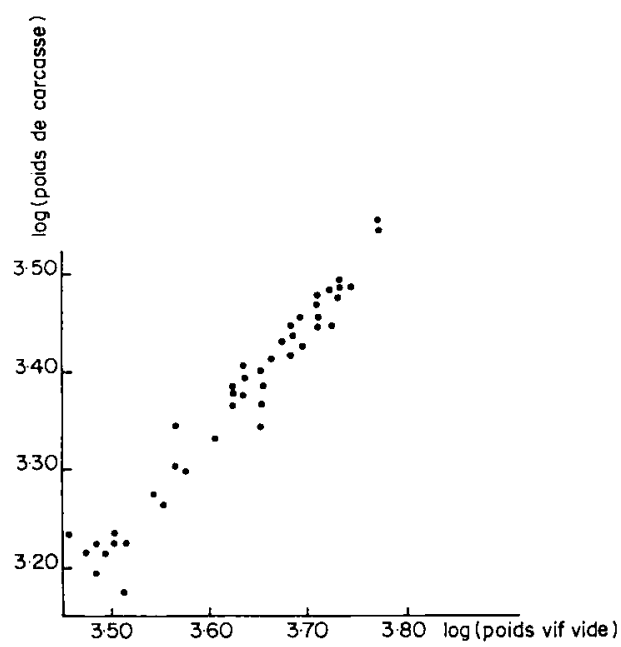

FIG. 2. - Evolution du poids de carcasse en fonction du poids vif vide chez le taurillon Frison de 9 à 15 mois

\section{RÉSULTATS}

\section{Modifications de la croissance relative entre 9 et 15 mois}

La croissance par rapport à l'ensemble de référence des différents tissus ou organes est relativement constante, tout au long de la période d'engraissement; en effet, le test đe curvilinéarité est toujours négatif (tabl. 2, 3, 4, 5) à l'exception des trois muscles $L$. dorsi, Psoas major et Pectorales profundi (tabl. 4); pour ceux-ci, l'équation curvilinéaire s'ajuste mieux que l'équation linéaire $(\mathrm{P}<0,0 \mathrm{I})$ aux données expérimentales, mais l'ajustement à 2 droites ne diminue pas significativement la variance résiduelle $(\mathrm{P}<\mathrm{O}, \mathrm{OI})$. Comme il apparait illusoire d'effectuer sur une si courte période, un ajustement à un plus grand nombre de droites (qui envelopperaient la courbe du second degré), nous pouvons considérer que le coefficient de régression linéaire est un indice de croissance relative moyen au cours de la période d'engraissement; le sens de l'évolution de cet indice est donné par le signe du terme du second degré de l'équation curvilinéaire (tabl. 4).

Croissance relative de la carcasse et des principaux organes par rapport à celle du corps vide de 9 à 15 mois (tabl. 2)

La carcasse a une croissance légèrement plus rapide $(P<0,05)$ que celle du cinquième quartier, ce qui se traduit par une augmentation du rendement en carcasse.

Parmi les éléments du cinquième quartier, ce sont les dépôts adipeux (périrénaux, péritonéaux, mésentériques et péricardiques) qui ont la plus forte croissance relative ( $b=2,26 \pm 0, \mathrm{II})$; les différents organes (cœur, poumons, tube digestif), la tête et le cuir ont un coefficient d'allométrie compris entre 0,60 et 0,90 tandis que celui des reins et des os canons est voisin de 0,50 . 
TABLEAU 2

Croissance relative des principaux éléments du corps vide $(\mathrm{Y})$

\begin{tabular}{|c|c|c|}
\hline & $b \pm s b\left({ }^{1}\right)$ & $F\left({ }^{2}\right)$ \\
\hline Carcasse & $1,04 \pm 0,02$ & 0,31 \\
\hline Muscles . . . . . . . . . . & $0,94 \pm 0,02$ & 0,80 \\
\hline Squelette $\ldots \ldots \ldots \ldots \ldots \ldots$ & $0,79 \pm 0,03$ & 0,21 \\
\hline Dépôts adipeux de la carcasse. & $1,98 \pm 0,08$ & 0,11 \\
\hline Dépôts adipeux du $5^{\mathrm{e}}$ quartier. & $2,26 \pm 0,10$ & 0,80 \\
\hline Dépôts adipeux totaux $\ldots \ldots$ & $2,05 \pm 0,08$ & 0,21 \\
\hline Foie $\ldots \ldots \ldots \ldots \ldots \ldots$ & $0,69 \pm 0,07$ & 0,32 \\
\hline Couur $\ldots \ldots \ldots \ldots \ldots \ldots \ldots$ & $0,83 \pm 0,04$ & 0,01 \\
\hline Reins $\ldots \ldots \ldots$ & $0,56 \pm 0,06$ & 0,14 \\
\hline Poumons .... & $0,73 \pm 0,09$ & 0,05 \\
\hline Cuir $\ldots \ldots \ldots \ldots$ & $0,83 \pm 0,05$ & 3,59 \\
\hline Tube digestif total & $0,74 \pm 0,04$ & 0,01 \\
\hline Rumen $\ldots \ldots \ldots \ldots \ldots \ldots$ & $0,89 \pm 0,08^{*}$ & 0,08 \\
\hline Feuillet $\ldots \ldots \ldots \ldots \ldots \ldots$ & $1,13 \pm 0,12^{*}$ & 1,13 \\
\hline Caillette...$\ldots$ & $0,60 \pm 0,09$ & 1,78 \\
\hline Intestins $\ldots \ldots$ & $0,54 \pm 0,05$ & 0,16 \\
\hline Tête ........ & $0,78 \pm 0,02$ & 0,59 \\
\hline Os canons ... & $0,50 \pm 0,04$ & 0,63 \\
\hline Testicules . . . . . . . . . . & $1,21 \pm 0,09$ & 0,98 \\
\hline
\end{tabular}

(1) Coefficient de régression de $\log \mathrm{Y}$ sur $\log \mathrm{X}$ calculé à partir de 65 animaux ( $\mathrm{X}$ : poids vif vide; $\mathrm{Y}$ : poids de l'élément considéré).

(2) Test de linéarité entre les variables $\log \mathrm{Y}$ et $\log \mathrm{X}$. La relation est linéaire $(P<0,01)$ pour $F<7,08$.

* : $b$ non significativement différent de $1(P<0,05)$.

\section{TABLEAU 3}

Croissance relative des principaux tissus de la carcasse

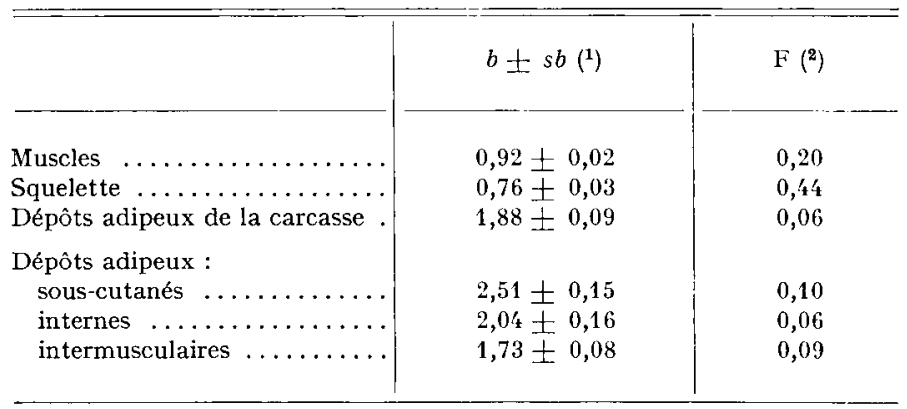

(1) Coefficient de régression de $\log \mathrm{Y}$ sur $\log \mathrm{X}$ calculé à partir de 65 animaux ( $\mathrm{X}$ : poids de la carcasse, $\mathrm{Y}$ : poids du tissu considéré).

(2) Test de linéarité entre les variables $\log \mathrm{Y}$ et $\log \mathrm{X}$. La relation est linéaire $(\mathrm{P}<0,01)$ pour $\mathrm{F}<7,08$. 
Les divers compartiments du tube digestif ont des croissances relatives très différentes, plus élevées pour le rumen et surtout le feuillet ( $b$ voisin de $I$ ), plus faible pour la caillette et les intestins ( $b$ voisin de 0,6$)$.

\section{Croissance relative des différents tissus de la carcasse}

par rapport à celle de la carcasse entière entre 9 et 15 mois (tab1. 3)

Le coefficient d'allométrie des dépôts adipeux est voisin de $2(b=\mathrm{I}, 88 \pm 0,09)$, tandis que ceux des muscles et du squelette sont significativement inférieurs à I (respectivement $0,92 \pm 0,02$ et $0,76 \pm 0,03$ ).

Parmi les dépôts adipeux de la carcasse, les dépôts sous-cutanés et internes ont une croissance nettement plus rapide $(b=2,5 \mathrm{I} \pm 0, \mathrm{I} 5$ et $b=2,04 \pm 0, \mathrm{I} 6$ respectivement) que celle des dépôts intermusculaires $(b=\mathrm{I}, 73 \pm 0,08)$.

\section{Croissance relative des principaux muscles des différentes régions corporelles par rapport à celle de la musculature totale entre 9 et 15 mois (tab1. 4)}

Les coefficients d'allométrie des muscles des membres sont voisins de l'unité. (T. brachï caput laterale, biceps femoris, tensor facia lata), où significativement supérieurs à I dans le cas des membres antérieurs ( $T$. brachï caput longum) ou significativement inférieurs à I dans le cas des membres postérieurs (semitendinosus, semimembranosus).

\section{TABLEAU 4}

Croissance relative des principaux muscles $(\mathrm{Y})$ des différentes régions corporelles, par rapport à celle de la musculature totale (X) (1)

\begin{tabular}{|c|c|c|c|c|c|}
\hline Régions & Muscles & $b+s b\left({ }^{1}\right)$ & $F\left({ }^{2}\right)$ & $\begin{array}{c}\text { Concavité } \\
\left.\qquad{ }^{3}\right)\end{array}$ & $\begin{array}{c}\text { Résultats de } \\
\left.\text { ButterFIELD ( }{ }^{4}\right)\end{array}$ \\
\hline $\begin{array}{c}\text { Membre } \\
\text { antérieur }\end{array}$ & $\begin{array}{l}\text { triceps brachii } \\
\text { caput longum } \ldots \ldots \ldots \\
\text { caput latérale } \ldots \ldots \ldots\end{array}$ & $\begin{array}{l}1,09 \pm 0,04 \\
0,91 \pm 0,06^{*}\end{array}$ & $\begin{array}{l}0,01 \\
0,9^{\prime} t\end{array}$ & & $\begin{array}{l}0,99 \pm 0,03 \\
0,90 \pm 0,01\end{array}$ \\
\hline $\begin{array}{c}\text { Membre } \\
\text { postérieur }\end{array}$ & $\begin{array}{l}\text { semitendinosus } \ldots \ldots \\
\text { semimembranosus }\end{array}$.... & $\begin{array}{l}0,88 \pm 0,06 \\
0,72 \pm 0,05 \\
0,93 \pm 0,04^{*} \\
0,92 \pm 0,06^{*}\end{array}$ & $\begin{array}{l}2,86 \\
1,46 \\
0,01 \\
0,06\end{array}$ & & $\begin{array}{l}0,95 \pm 0,09 \\
0,87 \pm 0,06 \\
0,96 \pm 0,04 \\
1,01 \pm 0,01\end{array}$ \\
\hline $\begin{array}{l}\text { Région } \\
\text { thoracique }\end{array}$ & $\begin{array}{l}\text { cutaneus trunci ........ } \\
\text { pectorales profundi } \ldots .\end{array}$ & $\begin{array}{l}1,07 \pm 0,08^{*} \\
0,98 \pm 0,03^{*}\end{array}$ & $\begin{array}{l}2,31 \\
7,9 / 4\end{array}$ & + & $\begin{array}{l}1,17 \pm 0,02 \\
1,00 \pm 0,01\end{array}$ \\
\hline $\begin{array}{l}\text { Région } \\
\text { dorsale }\end{array}$ & $\begin{array}{l}\text { L. dorsi } \ldots \ldots \ldots \ldots \\
\text { psoas major } \ldots \ldots \ldots\end{array}$ & $\begin{array}{l}0,91 \pm 0,04 \\
0,85 \pm 0,05\end{array}$ & $\begin{array}{r}32,32 \\
7,90\end{array}$ & - & $\begin{array}{l}0,90 \pm 0,05 \\
0,83 \pm 0,09\end{array}$ \\
\hline
\end{tabular}

* : $b$ non significativement différent de $1(\mathrm{P}<0,05)$.

(1) Coefficient de régression de $\log \mathrm{Y}$ sur $\log \mathrm{X}$ calculé à partir de 58 animaux.

$\left.{ }^{2}\right)$ Test de linéarité entre les variables $\log \mathrm{Y}$ et $\log \mathrm{X}$; la relation est linéaire si $\mathrm{F}<7,12(\mathrm{P}<0,01)$.

$\left.{ }^{3}\right)$ Signe du coefficient du terme en $(\log X)^{2}$ dans l'équation curvilinéaire (si le test de linéarité est négatif).

(4) Résultats de Butterfield (1966c) : phase 3. 
TABIEAU 5

Croissance relative de la chair (muscles + gras) des différentes régions corporelles (Y) par rapport à la croissance de la chair de la carcasse (X)

\begin{tabular}{|c|c|c|}
\hline & $b \pm s b(\mathbf{1})$ & $\mathrm{F}\left({ }^{2}\right)$ \\
\hline $\begin{array}{l}\text { Membre antérieur } \ldots \ldots \ldots \ldots \ldots \\
\text { Membre postérieur } \ldots \ldots \ldots \ldots\end{array}$ & $\begin{array}{l}1,02 \pm 0,03^{*} \\
0,79 \pm 0,02\end{array}$ & $\begin{array}{l}0,14 \\
0,23\end{array}$ \\
\hline Région cervicale $\ldots \ldots$ & $1,21 \pm 0,05$ & 0,18 \\
\hline $\begin{array}{l}\text { Région dorsale } \ldots \ldots \ldots \ldots \ldots \\
\text { Région lombaire } \ldots \ldots \ldots \ldots \ldots \\
\text { Région sacrée } \ldots \ldots \ldots \ldots \ldots \ldots \\
\text { Ensemble des régions dorso- } \\
\quad \text { lombo-sacrées } \ldots \ldots \ldots \ldots \ldots\end{array}$ & $\begin{array}{l}1,07 \pm 0,05^{*} \\
0,95 \pm 0,03^{*} \\
0,83 \pm 0,03 \\
0,97 \pm 0,02^{*}\end{array}$ & $\begin{array}{l}1,08 \\
0,01 \\
0,01 \\
0,02\end{array}$ \\
\hline $\begin{array}{l}\text { Régions thoracique et abdomi- } \\
\text { nale } \ldots \ldots \ldots \ldots \ldots \ldots \ldots \ldots\end{array}$ & $1,17 \pm 0,03$ & 2,05 \\
\hline
\end{tabular}

(1) Coefficient de régression de $\log \mathrm{Y}$ sur $\log \mathrm{X}$ calculé à partir de 51 animaux.

$\left({ }^{2}\right)$ Test de linéarité entre $\log \mathrm{Y}$ et $\log \mathrm{X}$. La relation entre les variables est linéaire $(\mathrm{P}<0,01)$ pour $\mathrm{F}<7,19$.

* : $b$ non significativement différent de $1(\mathrm{P}<0,05)$.

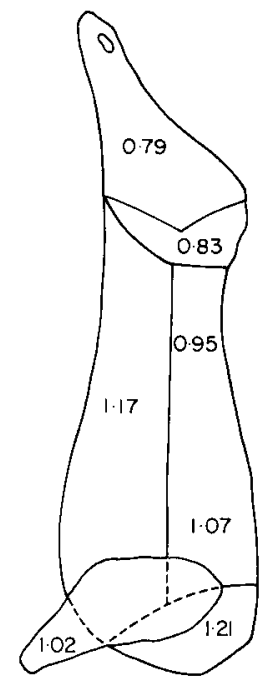

Fig. 3. - Coefficient d'allométrie de la chair (muscles + dépôts adipeux) des différentes régions corporelles chez le taurillon Frison de 9 à 15 mois 
La croissance relative des muscles de la poitrine et du thorax est légèrement supérieure ou voisine de celle de la musculature totale (cutaneus trunci, pectorales profundi), tandis que celle des muscles du dos ( $L$. dorsi, psoas major) est significativement plus faible. De plus, la croissance relative de ces derniers a tendance à diminuer au cours de la période d'engraissement (tabl. 4).

Croissance relative de la chair (muscles + dépôts adipeux) des différentes régions corporelles par rapport à celle de la chair de la carcasse totale de 9 à 15 mois (tab1. 5, fig. 3)

La région cervicale est avec les régions thoracique et abdominale, la zone dont la croissance relative est la plus élevée ( $b$ voisin de $I, 2)$; viennent ensuite par ordre décroissant, le membre antérieur $(b=\mathrm{I}, 02 \pm 0,03)$, l'ensemble des régions dorsolombo-sacrées $(b=0,97 \pm 0,02)$ et enfin le membre postérieur $(b=0,79 \pm 0,02)$. On observe également cette priorité croissante de 1'arrière vers l'avant au niveau des régions dorso-lombo-sacrées (région sacrée : $b=0,83 \pm 0,03$; région lombaire : $b=0,95 \pm 0,03$; région dorsale : $\mathrm{I}, 07 \pm 0,05)$.

\section{DISCUSSION}

Les expériences n'ayant pas été planifiées pour cette étude, nous devons émettre un certain nombre de restrictions sur la valeur intrinsèque des coefficients de régression que nous avons calculés.

Cette étude ne porte en effet que sur une courte période de la vie de l'animal, elle ne peut donc permettre de décrire de façon complète la croissance des taurillons, car d'après les travaux de BUTTERFIELD (I965), la plupart des modifications de la croissance relative des différents tissus interviennent dans le plus jeune âge. E.n outre, le gain de poids vif journalier des animaux a décru au cours de la période d'engraissement de I $398 \pm 293 \mathrm{~g}$ pendant la phase 9 - $\mathrm{I} 2$ mois à $997 \pm 250 \mathrm{~g}$ au cours de la phase I2-I5 mois. Toutefois, ce ralentissement de la croissance, bien qu'un peu fort, correspond à un phénomène physiologique habituel au cours de l'engraissement. Enfin, les niveaux d'alimentation ou les modes d'élevage différents, ont pu éventuellement influencer plus ou moins l'évolution de la composition des animaux.

Ces restrictions étant apportées, le nombre important des animaux utilisés, et leur homogénéité dans chaque lot nous permettent de penser que les coefficients de régression que nous avons obtenus, sont cependant un bon reflet de la croissance relative moyenne des différents tissus ou organes de ces animaux, entre 9 et I 5 mois.

Cette étude a ainsi permis de préciser les modifications de la composition corporelle des jeunes bovins mâles Frisons pendant la phase d'engraissement : augmentation du rendement en carcasse, augmentation importante de la part prise par les dépôts adipeux dans la carcasse, et particulièrement par les dépôts sous cutanés et internes, diminution de celle des muscles et surtout du squelette. Elle apporte des résultats nouveaux pour ce type d'animal, sur la croissance relative des différentes régions corporelles et de leurs muscles : la part prise par les régions antérieures et ventrales (membres antérieurs, régions cervicales, abdominale et thoracique) tend 
à augmenter, tandis que celle occupée par les parties postérieures et dorsales (régions dorsale, lombaire, sacrée, membre postérieur) tend à diminuer (fig. 3). On observe également ce phénomène au niveau de la croissance des principaux muscles de ces différentes régions.

A notre connaissance, il existe très peu de résultats sur la croissance relative des tissus ou organes chez le bovin mâle, mis à part ceux de HEDRICK et al. (I969) et de MukHoty et BERG (I97I). Nous sommes donc amenés à comparer nos résultats presqu'exclusivement à ceux qui ont été obtenus sur le bouf. Il n'y a pas de différences nettes entre taureaux et bœufs, dans la croissance relative de la carcasse, des différents éléments du cinquième quartier ou des muscles (MOULToN et al., I922 ; SEEBECK et TUlLOH, Ig68 $b$; ButTERFIELD et BERG, Ig66 a). De même, la hiérarchie de croissance relative des différents tissus de la carcasse est semblable : la part des muscles et du squelette dans la carcasse tend à diminuer, tandis que le pourcentage des dépôts adipeux augmente. Il semble cependant, d'après la comparaison établie au tableau 6 et les travaux de HEDRICK et al. (Ig69), que la croissance relative des

TABLEAU 6

Coefficients d'allométrie des tissus de la carcasse par rapport au poids de carcasse

\begin{tabular}{|c|c|c|c|}
\hline Auteurs & $\begin{array}{c}\text { Résultats } \\
\text { présentés } \\
\text { dans le texte }\end{array}$ & $\begin{array}{c}\text { SEEBECK } \\
\text { et Tulloh } \\
(1968 a \text { et } b)\end{array}$ & $\begin{array}{l}\text { Tulloh } \\
\text { (1963) }\end{array}$ \\
\hline Animaux (nombre, âge) & $\begin{array}{l}65 \text { taurillons } \\
9 \text { à } 15 \text { mois }\end{array}$ & $\begin{array}{l}10 \text { bceufs } \\
11 \text { a } 19 \text { mois }\end{array}$ & $\begin{array}{c}6 t_{ \pm} \text {boeufs } \\
-\end{array}$ \\
\hline $\begin{array}{c}\text { Coefficients par rapport } \\
\text { au poids de carcasse }\end{array}$ & & & \\
\hline Squelette ........... & 0,76 & 0,68 & 0,65 \\
\hline Muscles $\ldots \ldots \ldots \ldots \ldots$ & 0,92 & 0,83 & 0,85 \\
\hline $\begin{array}{c}\text { Dépôts adipeux (de la car- } \\
\text { casse) } \ldots \ldots \ldots \ldots \ldots \ldots\end{array}$ & 1,88 & 1,98 & 1,80 \\
\hline $\begin{array}{l}\text { Dépôts adipeux : } \\
\text { sous-cutanés } \ldots \ldots \ldots \ldots \\
\text { internes } \ldots \ldots \ldots \ldots \ldots \\
\text { intermusculaires } \ldots \ldots \ldots\end{array}$ & $\begin{array}{l}2,51 \\
2,04 \\
1,73\end{array}$ & $\begin{array}{l}2,58 \\
1,86(1) \\
1,61\end{array}$ & \\
\hline
\end{tabular}

(1) Ce chiffre concerne le gras interne de la carcasse + le gras périrénal.

dépôts adipeux soit plus élevée chez les bœufs que chez les taurillons de race Hereford, tandis que celle des muscles et du squelette sont légèrement inférieures. Cependant, Mukhoty et BERG (I97I) ne trouvent pas de différences significatives dans la croissance relative des tissus, entre les bœufs et les taureaux de différentes races anglosaxonnes. Bien que le classement des régions corporelles d'après leur croissance relative soit identique chez les bœufs et les taureaux (Moulton et al., I922; ANonyme, I966), ces derniers ont un développement plus rapide de la région cervicale. Ce 
niveau de croissance différent, résulte probablement de 1'effet des hormones sexuelles (BRADFIELD, I967); il entraîne une répartition différente des quartiers avant et arrière dans la carcasse (GEAY et MALTERRE, I97I).

Le résultat le plus important de cette étude nous paraît être la diminution au cours de la période analysée de l'importance relative des régions postérieure et dorsale, et notamment de la région lombaire $(b=0,95)$. Bien qu'en désaccord avec les lois classiques de l'École de Hammond, ce résultat concorde avec ceux des travaux de LutTingh (ig62), ANonyme (I966), ButTerfield et Berg (Ig66 b), SeEBECK TUl,LOH (I968) et avec ceux calculés par HEDRICK (Ig66) à partir des travaux de Moulion et al. (I922). LuITINGH (I962) pense que cette contradiction provient de ce que, dans la découpe pratiquée par l'École de Hammond, la région lombaire comprenait des parties de muscles appartenant à la paroi abdominale, muscles à croissance très rapide (BUT'TERFIELD et BERG, Ig66 $a$ et $b$; SEEBECK et TULLOH, Ig68a).

Cette première description de la croissance relative des taurillons Frisons pendant la phase d'engraissement doit être prolongée maintenant par une étude cinétique portant sur une plus longue période, comportant un plus grand nombre de stades d'abattage, afin de mettre en évidence, si elle existe, une évolution dans la croissance relative des différents tissus adipeux. Ceci permettrait de déterminer avec précision à quel stade physiologique certains se développent prioritairement et de tenter alors, à ces stades, de modifier l'évolution de la composition corporelle. En ce qui concerne l'étude du développement musculaire, il faudrait diviser la musculature en régions anotomiquement bien définies (cf. travaux de BERG, BUTTERFIELD, SEEBECK, TULLOH). Enfin, il serait intéressant de compléter cette étude du développement des tissus et organes, par une étude sur l'évolution de la composition chimique de la carcasse et du corps entier.

Rę̧u pour publication en avril 1974.

\author{
SUMMARY \\ RELATIVE GROWTH OF THE DIFFERENT ORGANS, CARCASS TISSUES \\ AND CARCASS JOINTS IN FRIESIAN BULLS DURING THE PERIOD \\ OF FATTENING FROM 9 TO I5 MONTHS
}

A total of 65 young entire bovine males of the French Friesian breed were slaughtered between 9 to 15 months of age, and subsequently dissected. The relative growth of the main components of the animal has been described utilizing the allometric equation of Huxley.

For the weight of most of the organs, the allometric coefficient, relative to empty body weight was less than $\mathrm{I}$, whereas that of the total fat depots was close to 2 and that of dressed carcass was slightly greater than I. The magnitude of the relative growth coefficients of the dressed carcass tissues was comparable to that already reported for other types of bovines and for other species : skeleton $(b=0,76)$, muscles $(b=0,92)$, total carcass fat $(b=\mathrm{I}, 58)$; of the carcass fat, the subcutaneous fat showed the highest relative growth $(b=2,5 \mathrm{I})$. Regarding the relative growth of the various joints of the carcass, it was shown that the proportions of hind and back regions tend to decrease during fattening period from 9 to 15 months, whereas those of the anterior and abdominal regions tend to increase. 


\section{RÉFÉRENCES BIBLIOGRAPHIQUES}

Anonyme, I966. A comparison of the growth of different types of cattle for beef production. Report of Major Beef Research Project. The Royal Smithfield Club, London.

Benevent M., I97I. Croissance relative pondérale post natale, dans les deux sexes, des principaux tissus et organes de l'agneau Merinos d'Arles. Ann. Biol. anim. Bioch. Biophys., 11, 5-39.

Bradfield P. G. E., 1967. Sex differences in the growth of sheep in Growth and development of mammals, 92-I08. Butterworths, London.

BUTteRfield R. M., r963. Relative growth of the musculature of the ox. Carcass composition and appraisal of meat animals, 7, I-I4 (Ed. D. E. TRIBE). East Melbourne, C. S. I. R. O.

Butterfield R. M., 1965. Practical implications of anatomical Research in beef-cattle. Proc. N.Z. Soc. Anim. Prod., 25, I52-162.

Butterfield R. M., Berg R. T., rg66a. A classification of bovine muscles, based on their relative growth patterns. Res. Vet. Sci., $7,326-332$.

Butterfield R. M., Berg R. T., I $966 \mathrm{~b}$. Relative growth patterns of commercially important muscle groups of cattle. Res. Vet. Sci., 7, 389-393.

Butterfield R. M., Berg R. T., Ig66 $c$. A nutritional effect on relative growth of muscles. Proc. Austr. Soc. An. Prod., 6, 298-304.

ButTERfield R. M., Johnson E. R., 1967. The effect of growth rate of muscle in cattle on conformation as influenced by muscle-weight distribution, in Growth and development of mammals, 212-223. London, Butterworths.

Butterfield R. M., Johnson E. R., I97I. A study of growth in calves. II. Relative growth in muscles. J. agric. Sci., 76, 457-458.

Butterfield R. M., Pryor W. I., Berg R. T., r966. A study of carcass growth in calves. Res. vet. Sci., $4 \mathrm{I} 7-423$.

Cantier J., Vezinhet A., Rouvier A., Dauzier L., 1969. Allométrie de croissance chez le Lapin. I. Principaux organes et tissus. Ann. Biol, anim. Bioch. Biophys., 9, 5-39.

Charlet P., Dumont B. L., I953. Découpe du bœuf. In Production et Commercialisation de la Viande. Comptes rendus des troisièmes journées de la F. E. Z. (p. rog). Sienne-Rome, septembre r953.

Geay Y., Malterre C., I97x. Influence de la castration et de la nature des glucides de la ration sur la croissance et la qualité des carcasses de bovins abattus à 24 mois. Ann. Zootech., 20, 25 I-257.

Huxley J. S., I932. Problems of relative growth. Methuen, London.

Hedrick H. B., Ig66. Beef carcass composition. North Central Regional Publication. Regional Project N. C. 58 .

Hedrick H. B., Thompson G. B., Kranse G. F., 1969. Comparison of feedlot performance and carcass characteristics of half-sib bulls, steers and heifers. J. Anim. Sci., 29, 687-694.

Luitingh H. C., I962. Developmental changes in beef steers as influenced by fattening, age and type of ration. J. agric. Sci., 58, I-46.

Moulton R., Trowbridge P. F., Haigh L. D., r922. Studies in animal nutrition. II. Changes in proportions of carcass and offal on different planes of nutrition. Mo. Agr. Expt. Station, Research Bulletin $n^{\circ} 54$.

Muкhoty H., Berg R. T., I97r. Influence of breed and sex on the allometric growth patterns of major bovine tissues. Anim. Prod., 13, $219-227$.

PaAlson H., 1955. Conformation and body composition. In Progress in the physiology of farm animals, 2, 430 (Butterworth, London).

Rhodes D. N., 1969. Meat Production from entire male animals. London. J. A., Churchill LTD.

SEEBECK R. M., I967. Developmental growth and body weight loss of cattle. I. Experimental design, body weight growth, and the effects of developmental growth and body weight loss on the dressed carcass and the offal. Aust. J. Agric. Res., 18, xor5-31.

Seebeck R. M., Tulloh N. M., rg68 $a$. Developmental growth and body weight loss of cattle. II. Dissected components of the commercially dressed and jointed carcass. A ust. J. A gric. Res., 19, 477-95.

Seebeck R. M., TUlloh N. M., I968 b. Developmental growth and body weight loss of cattle. III. Dissected components of the commercially dressed carcass following anatomical boundaries. Aust. $J$. A gric. Res., 19, $683^{-88}$.

Tullor N. M., I963. The carcass composition of sheep, cattle and pigs as function of body weight. In Carcass composition and appraisal of meat animal, 5, I-I6 (Éd. D. E. TRIBE) East Melbourne C. S. I. R. O.

Vezihnet A., Rouvier R., Dulor P.-P., Cantier J., 1972. Allométrie de croissance chez le Lapin. III. Principales régions du système musculaire. Ann. Biol. anim. Bioch. Biophys., 12, 33-45. 